\title{
Model-Based Classification of Polarimetric SAR Sea Ice Data
}

\author{
B. Scheuchl ${ }^{1}$, I. Hajnsek ${ }^{2}$ and I.G. Cumming ${ }^{1}$ \\ ${ }^{1}$ Dept. of Electrical and Computer Engineering, University of British Columbia, 2356 Main Mall, Vancouver, B.C., Canada \\ V6T1Z4 E-mail: bernds@ece.ubc.ca, ianc@ece.ubc.ca \\ ${ }^{2}$ German Aerospace Centre, Institute for Radio Frequency Technology and Radar Systems, Department SAR Technology, \\ P.O. Box 1116, 82234 Wessling, GermanyＥ-mail: irena.hajnsek@dlr.de
}

\begin{abstract}
This paper discusses the role of scattering decomposition models in the classification of polarimetric SAR sea ice data. The iterative Wishart classifier was applied to 3-frequency airborne SAR data acquired in the Beaufort Sea, and the scattering models were found to be helpful in interpreting the assigned classes. In addition to using the full data set, reduced data sets based on an eigenvector decomposition were investigated for their potential for classification, as the eigenvectors provided a separation of scattering mechanisms. The surface scattering component was found to be the dominant one for this data set, and yielded a classification similar to the full data set.
\end{abstract}

\section{INTRODUCTION}

Salt content, ocean currents and temperatures cause the sea ice in the polar oceans to have wide spatial and temporal variability. Spaceborne remote sensing has proven to be an important tool to monitor the extent and type of ice cover.

Polarimetric SAR sensors provide a more complete inference of surface parameters than is possible with a singlechannel radar system. It has been shown that different scattering mechanisms can be extracted using scattering decomposition techniques. Scattering decomposition in combination with a Bayesian minimum distance classifier allows an unsupervised classification, and has been successfully used for sea ice classification [1]. As the classes obtained by the classification do not directly give a physical interpretation of the scatterer, a manual step is needed to interpret the results. Using the polarimetric parameters of the class means are one way of making this task easier, but they still do not allow a physical interpretation of the scatterer. The main problem with the Wishart classifier lies in the strong dependence of the amplitude of the complex scattering matrix. However, a physical interpretation of the resulting classes is still needed, and can be obtained from the polarimetric information content.

In this paper we investigate two model-based ways of utilising radar scattering information to reduce the polarimetric information content and to interpret the classes obtained by the Wishart classifier.

\section{Airsar Polarimetric Data}

In March 1988, the JPL airborne AIRSAR acquired fully polarimetric SAR data of sea ice in the Beaufort Sea using three frequencies, C-, L-, and P-band. The acquisition was part of a larger study including ground measurements. The test site is characterised by multi-year ice (MYI) with floe thickness' of up to $6 \mathrm{~m}$; compressed first year ice (FYI) surrounding the floes; smooth and ridged FYI cover the rest of the area. Ice motion frequently opens up the clods, but thin new ice covers the open part almost instantly due to the cold temperatures. The average thickness of the FYI near the ice camp was reported to be 1.5-m [2].

\section{SCATTERING DECOMPOSITION}

To investigate of the sea ice properties with respect to the three frequencies and to the scattering mechanisms, a model based and an eigenvector-based decomposition has been applied to the AIRSAR data set (scene 1372).

\section{A. Freeman-Durden decomposition}

The Freeman-Durden decomposition [3] of the scattering mechanisms is a helpful tool for the interpretation of the scatterer, based on a physical model. The decomposition uses simple scattering processes to model the scattering behaviour of vegetated terrain. According to this model, backscattering from vegetated terrain can be regarded as the superposition of three single scattering processes: surface, dihedral and volume scattering. These components can be separated, and Fig. 1 gives the surface scattering and volume contribution of the sea ice for the different frequencies. Surprisingly, the contribution of dihedral scattering in all frequencies is very small, as ice ridges can create a dihedral effect. The main scattering contribution is coming from the surface scattering, which is observed in all frequencies, and the C-band data has the most balanced energy between surface and volume scattering throughout the scene (as shown by the ratios in Fig. 2). Since the volume contribution usually increases with increasing wavelength, it is surprising that the surface scattering contribution is much higher for L- and P-band.

This fact can be explained by the ground measurements. The big floes of the MYI contain a balanced proportion of surface and volume contribution in C-band, while in L- and P-band, both mechanisms are reduced due to the high penetration of the radar energy through the ice (causing reflections off the subsurface water-ice interface). As the interface is very smooth and non-conducting, surface and forward scattering mechanisms are predominantly present. Fig. 2 shows the ratio between surface and volume scattering - high ratios tend to represent equal contributions from surface and volume scattering, while the surface scattering dominates for low ratios. Due to the higher dielectric constant and the 
greater roughness of the compressed and ridged FYI (which can lie in the region of the $70-\mathrm{cm}$ P-band wavelength), a higher volume contribution is observed for this wavelength.
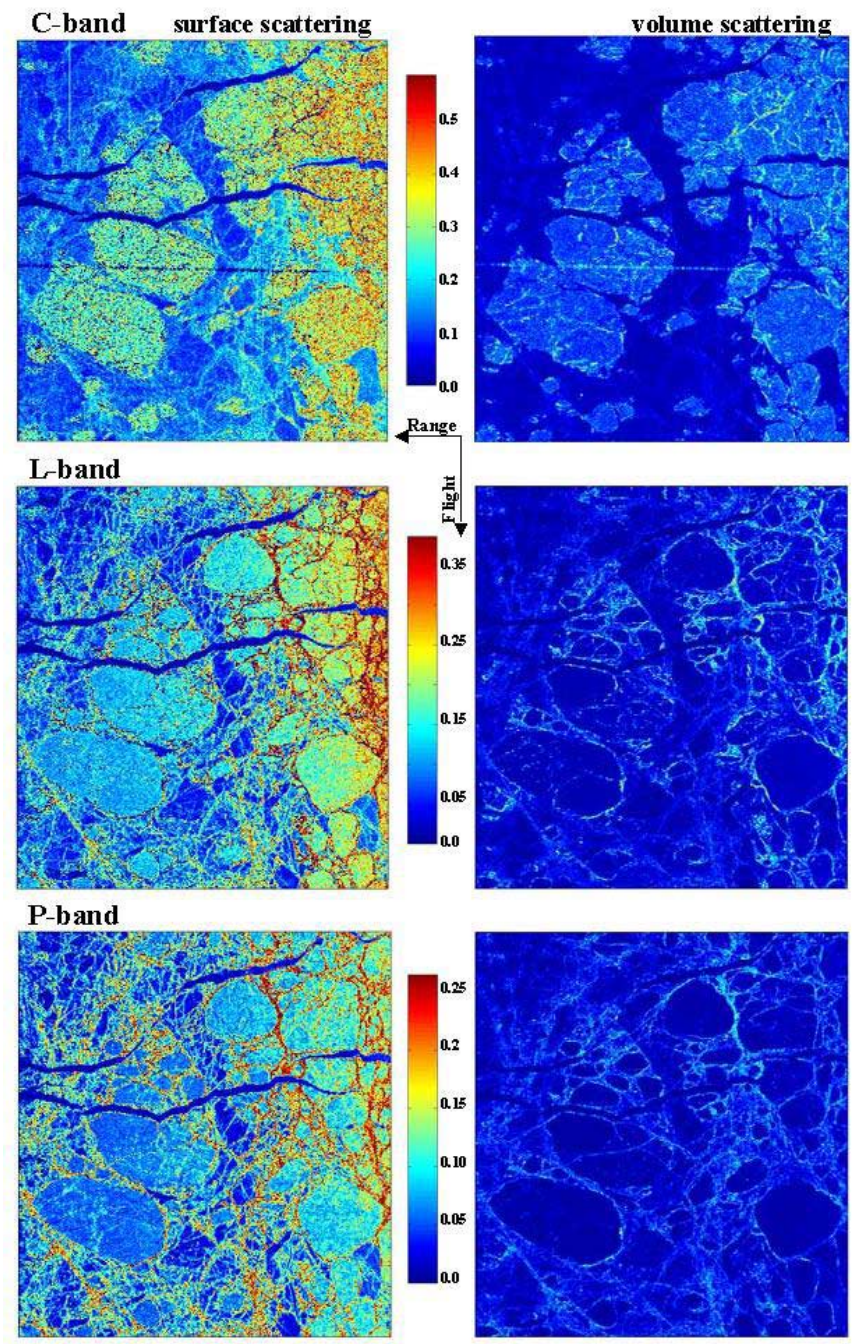

Fig. 1: Total power images of the Freeman-Durden decomposed C-, L- and P-band data (left: surface scattering, right: volume scattering)
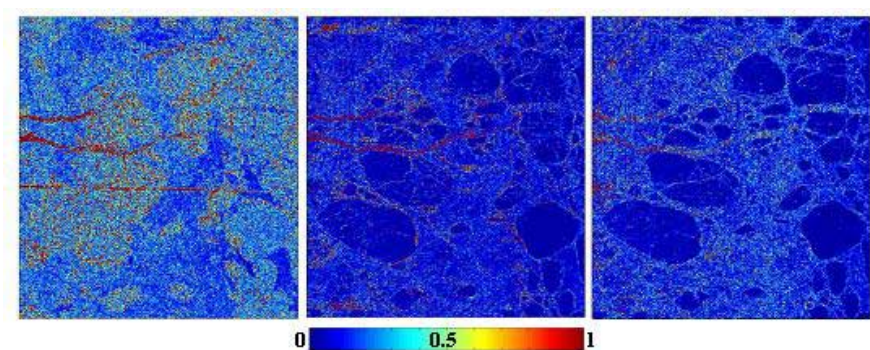

Fig. 2: Ratio images of surface to volume scattering using the FreemanDurden decomposition (1: C-band, 2: L-band, 3: P-band)

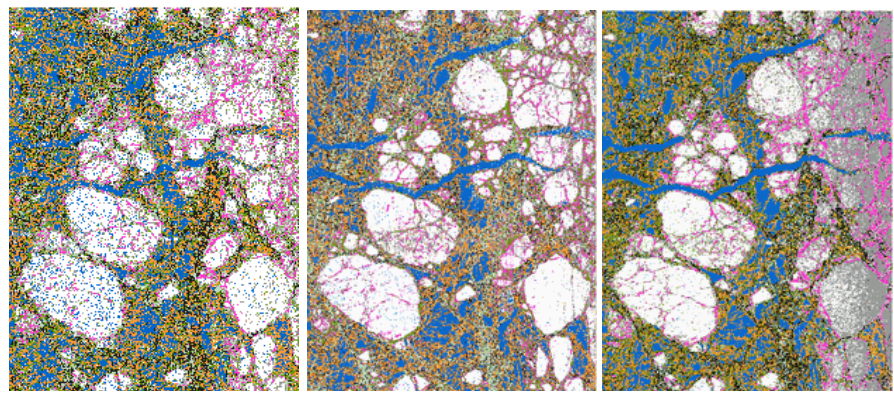

Fig. 3. Classification results after 12 iterations and manual assignment of classes to ice types. (1: $\left.\alpha \_\max , 2: \alpha \_\operatorname{mid}, 3: \alpha \_\min \right)$

\section{B. Eigenvector based decomposition}

Another way of extracting scattering mechanisms is to use an eigenvector decomposition of the coherency matrix [4]. Each eigenvector can be interpreted as a scattering matrix of one part of the received energy, and the contribution is proportional to the size of the corresponding eigenvalue. The eigenvector can be expressed using four angles, one of which, the so-called $\alpha$-angle, is usually taken to be representative of the average scattering mechanism [5]. For this study, the $\alpha-$ angle for each eigenvector is computed, which means that three scattering mechanisms can be extracted for each pixel -the eigenvectors with the minimum, middle, and maximum $\alpha$-angle. Only the minimum $\alpha$-angle can be interpreted as surface scattering with certainty (values for C-band data average $14^{\circ}$ with a standard deviation of $7^{\circ}$ ).

The decomposition was performed for all three frequencies, resulting in $\alpha \_$min, $\alpha \_$mid $\alpha \_$max scattering matrices for each frequency for each pixel. A multi-frequency Wishart classification can be performed for each scattering mechanism, i.e. for each $\alpha$, and the results are shown in Fig. 3. The ice types are described in Table I. Table II shows the confusion matrices for the ice types relative to the solution of the full data set used. This solution is discussed in more detail in [6].

TABLE I

Class Assignment To Ice Types

\begin{tabular}{ccc}
\hline Class & Colours & Description \\
\hline ThI / SFYI & blue & $\begin{array}{c}\text { New forming thin ice / } \\
\text { Smooth first year ice }\end{array}$ \\
\hline RFYI / R & $\begin{array}{c}\text { orange } \\
\text { green } \\
\text { black }\end{array}$ & $\begin{array}{c}\text { Ridged first year ice / } \\
\text { Rubble }\end{array}$ \\
\hline CFYI & pink & Compressed First Year Ice \\
& pastel green & Multi year Ice \\
\hline MYI & white & \\
& gray & \\
\hline
\end{tabular}


The iterative Wishart classification for all three $\alpha$ mechanisms converges at approximately the same rate as the classification using the full data set. A difference of less then $2 \%$ of pixels changing class per iteration can be noted if the convergence of single mechanisms and the full data set are compared.

The surface component ( $\alpha$-min) shows good agreement with the reference solution. A $\bar{n}$ incidence angle effect can be noted that is probably caused by a decreasing surface component with increasing incidence angle, which is also observed in the amplitude images. This observation supports the assumption that the eigenvector with the minimum $\alpha$ can be interpreted as surface scattering component.

The scattering component represented by $\alpha \_$mid tends to confuse the different FYI types relative to the full data set. A similar observation can be made for the $\alpha$ max component. The least confusion compared to the full data set can be observed for thin first year ice and multi-year ice.

Surface scattering dominates this particular data; therefore $\alpha$ min is the most likely candidate for a selected classification. Focus on a single scattering component seems advantageous as data models can be simplified.

TABLE II

Confusion Matrices For Different SCATtering Mechanisms

\begin{tabular}{crrrr}
\hline & ThI / SFYI & RFYI / R & \multicolumn{1}{l}{ CFYI } & \multicolumn{1}{l}{ MYI } \\
\hline $\boldsymbol{\alpha}$-max & & & & \\
ThI / SFYI & $\mathbf{7 0 . 8 1 \%}$ & $9.94 \%$ & $0.01 \%$ & $5.52 \%$ \\
RFYI / R & $26.25 \%$ & $\mathbf{7 5 . 2 9 \%}$ & $17.45 \%$ & $7.12 \%$ \\
CFYI & $0.00 \%$ & $5.60 \%$ & $\mathbf{6 9 . 8 7 \%}$ & $2.22 \%$ \\
MYI & $2.94 \%$ & $9.17 \%$ & $12.67 \%$ & $\mathbf{8 5 . 1 4 \%}$ \\
\hline $\boldsymbol{\alpha}$-mid & & & & \\
ThI / SFYI & $\mathbf{8 7 . 9 6 \%}$ & $8.77 \%$ & $0.00 \%$ & $1.00 \%$ \\
RFYI / R & $9.50 \%$ & $\mathbf{6 3 . 7 7 \%}$ & $35.85 \%$ & $2.85 \%$ \\
CFYI & $0.15 \%$ & $21.38 \%$ & $\mathbf{4 8 . 0 3 \%}$ & $3.52 \%$ \\
MYI & $2.39 \%$ & $6.08 \%$ & $16.12 \%$ & $\mathbf{9 2 . 6 3 \%}$ \\
\hline $\boldsymbol{\alpha}$-min & & & & \\
ThI / SFYI & $\mathbf{9 3 . 9 4 \%}$ & $5.89 \%$ & $0.00 \%$ & $0.29 \%$ \\
RFYI / R & $4.90 \%$ & $\mathbf{8 7 . 4 4 \%}$ & $14.67 \%$ & $4.74 \%$ \\
CFYI & $0.00 \%$ & $3.76 \%$ & $\mathbf{8 1 . 5 4 \%}$ & $3.44 \%$ \\
MYI & $1.16 \%$ & $2.91 \%$ & $3.79 \%$ & $\mathbf{9 1 . 5 3 \%}$ \\
\hline
\end{tabular}

\section{CONCLUSIONS}

Scattering decomposition allows the separation of different scattering mechanisms in fully polarimetric SAR data. Eigenvector based decomposition yields a scattering matrix for each of the mechanisms. This in turn allows the generation of a coherency matrix, which can be used, for Wishart classification. This paper addressed the problem of the lack of physical interpretability of the classes resulting from the Wishart classification. Two decomposition methods have been chosen to investigate the properties of sea ice.

The Freeman-Durden decomposition, which gives a 3component indication of scattering mechanisms, and the eigenvector decomposition, which separates scattering mechanisms by $\alpha$ angle, is studied. The Wishart classifier is applied for each $\alpha$ angle, and the results are compared to the classes obtained using the full data set. Using this reference the scattering mechanism represented by the minimum $\alpha$ yields the most similar classification result. The next challenge is to find a suitable clustering procedure, without losing the physical content of the classes.

\section{Acknowledgements}

The authors thank NASA/JPL for providing data. This work was partly funded by MacDonald Dettwiler and Associates under contract to the Canadian Space Agency (No. 9F028-0-4902/06) as part of the Earth Observation Applications Development Program.

\section{REFERENCES}

[1] B. Scheuchl, R. Caves, I.G. Cumming, and G. Staples, "Automated Sea Ice Classification Using Spaceborne Polarimetric SAR Data," in Proc. IGARSS'01, Sydney, July 2001.

[2] E. Rignot, and M.R. Drinkwater, "Winter sea-ice mapping from multiparameter synthetic-aperture radar data," Journal of Glaciology, vol. 40, no. 136, pp. 31-35, 1994.

[3] T. Freeman, and S.L. Durden, "A Three-Component Scattering Model for Polarimetric SAR Data," IEEE Trans. Geosci. and Remote Sensing, vol. 36, no. 3, pp. 963-973, May 1998.

[4] S.R. Cloude and E. Pottier, "An Entropy Based Classification Scheme for Land Applications of Polarimetric SAR," IEEE Trans. Geosci. and Remote Sensing, vol. 35, no. 1, pp. 68-78, January 1997.

[5] S.R. Cloude, E. Pottier, and W.M Boerner, "Unsupervised Image Classification Using the Entropy/Alpha/Anisotropy Method in Radar Polarimetry," Proceedings of 2002 AIRSAR Earth Science Applications Workshop, March 4-6, Pasadena, CA, http://airsar.jpl.nasa.gov

[6] B. Scheuchl, I. Hajnsek, I.G. Cumming, "Sea Ice Classification Using Multi-Frequency Polarimetric SAR Data," in Proc. IGARSS'02, Toronto, June 2002 (these proceedings). 